\title{
Relación entre la compactación y la succión en presas de tierra con suelos tipo $\mathrm{CH}$
}

\author{
Relationship between compaction and suction in earth dams with $\mathrm{CH}$ type soils
}

\section{Isaida Flores, Jenny García y Yoermes González}

Facultad de Ingeniería Civil, Universidad Tecnológica de La Habana José Antonio Echeverría, Calle 114 entre Ciclovía y Rotonda, Marianao,La Habana, Cuba, isaidafb@civil.cujae.edu.cu, jenny@civil.cujae.edu.cu, yoermes@civil.cujae.edu.cu

La construcción de presas de tierra ha puesto a la estabilidad de taludes en un plano de importancia ingenieril de primer orden, tanto por el aspecto económico como por las consecuencias indeseables debidas a sus fallos. El objetivo de esta investigación consiste en realizar un análisis para definir la dependencia de la curva de conductividad hidráulica del grado de compactación del suelo. El caso de estudio es una presa de tierra de $22 \mathrm{~m}$ de altura con prisma de drenaje, considerando los principios de la mecánica de suelos parcialmente saturados para los estados de final de construcción y operación de la misma, con tres suelos en la cortina de tipo CH y tres suelos arenosos en la cimentación. Para la ejecución de los modelos se utilizan las herramientas SIGMA/W, SEEP/W y SLOPE/W del programa GeoStudio 2012. Los principales resultados obtenidos muestran que la variación en la estabilidad se incrementa cuando el grado de compactación y la succión correspondiente oscilan entre 80 y 95\% del ensayo Proctor Estándar. Se concluye que resulta más efectivo emplear curvas características obtenidas para grados de compactación iguales o superiores al 95\%.

Palabras clave: suelos parcialmente saturados, estabilidad de taludes, relación de vacios, compactación
The construction of earth dams has placed the stability of slopes in a plane of engineering importance of the first order, both for the economic aspect and for the undesirable consequences due to their failures. The objective of this research is to perform an analysis to define the dependence of the hydraulic conductivity curve on the degree of soil compaction. The case study is a 22 $m$ high earth dam with a drainage prism, considering the principles of partially saturated soil mechanics for the final stages of construction and operation of the same, with three floors in the curtain of type $\mathrm{CH}$ and three sandy soils in the foundation. The SIGMA/W, SEEP/W and SLOPE/W tools of the GeoStudio 2012 program are used for the execution of the models. The main results obtained show that the variation in stability increases when the degree of compaction and the corresponding suction oscillate between 80 and 95\% of the Standard Proctor test, for which it is concluded that it is more effective to use characteristic curves obtained for compaction degrees equal to or greater than $95 \%$.

Keywords: partially saturated soils, slope stability, void ratio, compaction

\section{Introducción}

La Mecánica de Suelos inicialmente se desarrolló para estudiar los suelos saturados, condición que resulta extrema ya que se considera que la masa de suelo sólo se encuentra sometida a dos fases: sólida y líquida, y sus limitaciones son evidentes cuando se necesita explicar las deformaciones en suelos parcialmente saturados o de las estructuras que estos soportan.

Teniendo en cuenta su origen, los suelos parcialmente saturados pueden ser naturales o artificiales, dentro de los suelos artificiales se encuentran los suelos compactados, extensamente utilizados en obras de tierra (presas, terraplenes, etc.), que debido a su naturaleza son suelos parcialmente saturados (e.g. Fredlund y Rahardjo, 1993; Tristá, 2015; Musso y Suazo, 2019). Los suelos parcialmente saturados se encuentran sobre el nivel freático y pueden llegar incluso a estar secos en zonas cercanas a la superficie. El suelo en condiciones parcialmente saturadas se encuentra constituido por tres fases: sólida, líquida y gaseosa. 


\section{Succión en suelos parcialmente saturados}

Un suelo parcialmente saturado tiene la tendencia a recibir agua y aumentar su grado de saturación, si el suelo tiene determinado grado de saturación se requiere cierta fuerza para remover el agua del espacio poroso y disminuir el grado de saturación del suelo, estos efectos se pueden explicar mediante la succión (Fredlund y Rahardjo, 1993; Tristá, 2015). La succión en suelos no saturados está compuesta por la succión matricial y la succión osmótica y la suma de ambos componentes se denomina succión total. La curva característica del suelo es una relación entre la cantidad de agua en el suelo y la succión. La obtención de las succiones puede ser mediante distintos métodos y procedimientos que se dividen en directos e indirectos. El intervalo de medida de las succiones está en relación al tipo de equipo utilizado, así como del contenido de humedad presente, o si son utilizadas muestras inalteradas o remoldeadas durante el ensayo. El método del papel de filtro es un método indirecto que mide la succión total y matricial a partir de determinar el potencial del agua en el suelo por medio del potencial del agua retenida en un medio poroso llamado papel de filtro. Este método es muy utilizado porque las características del ensayo permiten su fácil aplicación en el laboratorio.

La curva característica del suelo es una relación entre la cantidad de agua en el suelo y la succión. La cantidad de agua en el suelo generalmente se cuantifica en términos de humedad gravimétrica $\omega$, grado de saturación $S$ o humedad volumétrica (Fredlund, 2003). A partir de la curva característica experimental, es posible obtener la curva de conductividad hidráulica del suelo. A diferencia de un suelo saturado, el cual mantiene una conductividad hidráulica constante; en un suelo no saturado, la conductividad hidráulica es variable, y depende del contenido de agua o estado de succiones que exista en el suelo (Mendoza, 2018) grados de saturación, velocidades de flujo, fuerzas de filtración, cargas hidraúlicas y presiones de poro. Fuerzas de filtración actúan en las partículas de suelo cada vez que un gradiente hidráulico genera flujo de agua. Estas fuerzas pueden afectar por erosión interna a suelos internamente inestables, ya que pueden producir el movimiento de la fracción más fina del suelo a través del esqueleto grueso del mismo suelo (Moffat, 2015).

Van Genuchten (1980) desarrolló una ecuación para obtener la curva de conductividad hidráulica del suelo, a partir de los parámetros obtenidos del ajuste de la curva característica del suelo parcialmente saturado, como se muestra en las fórmulas (1) y (2).

$$
K_{r}=\frac{\left[1-\left(\alpha S_{t}\right)^{\eta-1}\left\{1+\left(\alpha S_{t}\right)^{\eta}\right\}^{-m}\right]^{2}}{\left[1+\left(\alpha S_{t}\right)^{\eta}\right]^{m / 2}}
$$

Donde $K_{r}$ es el coeficiente de conductividad hidráulica no saturado del suelo, $S_{t}$ es la succión total, $\alpha, \eta, m$ son parámetros de ajuste $(m=1-1 / \eta)$.

$$
K=K_{\text {sat }} K_{r}
$$

Siendo $K$ y $K_{\text {sat }}$ los coeficientes de permeabilidad no saturado y saturado del suelo, respectivamente. Este método está incluido en los programas computacionales, lo que posibilita la estimación directa de la curva de conductividad hidráulica con la introducción de los parámetros obtenidos experimentalmente.

\section{Resistencia al corte de los suelos parcialmente saturados}

La envolvente de falla extendida para los suelos parcialmente saturados toma la siguiente forma matemática (Fredlund et al., 1978; Fredlund y Rahardjo, 1993; Tristá, 2015; Alanís, 2012).

$\tau=c^{\prime}+\left(\sigma_{n}-u_{a}\right) \tan \phi^{\prime}+\left(u_{a}-u_{w}\right) \tan \phi^{b}$

Donde $u_{a}$ es la presión del aire, $\left(\sigma_{n}-u_{a}\right)$ es el esfuerzo neto, $\left(u_{a}-u_{w)}\right.$ es la succión en el suelo, $c^{\prime}$ es la cohesión efectiva, $\phi^{\prime}$ y $\phi^{b}$ son el ángulo de fricción interna efectivo y el ángulo de succión, respectivamente. Los parámetros de resistencia para los suelos parcialmente saturados se ven afectados por la influencia de la succión y estos se pueden obtener a partir de los parámetros de resistencia del suelo saturado (Fredlund y Rahardjo, 1993; Tristá 2015). Como una alternativa para sustituir el uso de $\phi^{b}$, en SLOPE/W se implementa la fórmula propuesta por Vanapalli et al. (1996).

$$
\tau=c^{\prime}+\left(\sigma_{n}-u_{a}\right) \tan \phi^{\prime}+\left(u_{a}-u_{w}\right)\left[\left(\frac{\theta_{w}-\theta_{r}}{\theta_{s}-\theta_{r}}\right) \tan \phi^{\prime}\right]
$$

Donde $\theta_{w}, \theta_{s}$ y $\theta_{r}$ son el contenido de agua volumétrico, saturado y residual, respectivamente. La fuerza cortante 
no saturada de un suelo se puede estimar en función de la curva característica del suelo y de los parámetros de resistencia al corte saturados del mismo.

\section{Presas de tierra y estabilidad de taludes}

Las presas de tierra son obras de ingeniería construidas para almacenar y distribuir las reservas de agua con el objetivo de obtener beneficios económicos y sociales. En Cuba la construcción de presas de tierra también ha evolucionado significativamente. Actualmente es posible contar con 241 embalses a lo largo de toda la Isla. Para establecer el diseño y construcción de una presa, es necesario considerar factores económicos imprescindibles, así como el alcance social y el objetivo de la misma. Armas y Horta (1987) definieron algunos de los parámetros de diseño, que son las bases de la geometría utilizada en esta investigación.

El drenaje se utiliza para evitar que el agua infiltrada transporte el material de la presa, provocando así su destrucción. El tipo de dispositivo de drenaje puede variarse en diferentes áreas de la presa y su construcción debe seleccionarse sobre la base de la comparación técnicoeconómica de las diferentes variantes. En la presente investigación se plantea un drenaje de tipo prisma, dado que es el más utilizado en Cuba.

(Molina et al., 2017) contabilizaron los fallos en presas de tierra en Cuba, luego de realizar visitas a las empresas de aprovechamiento hidráulico de todas las provincias del país durante el año 2016, identificando un total de 52 casos. En base a estos estudios, el orden actual de ocurrencia de las causas más comunes de falla en presas de tierra en Cuba, difiriendo ligeramente con respecto al resto del mundo son: deslizamiento de taludes, rebase de la cortina, sifonamiento, pérdidas por filtraciones y agrietamiento.

Un talud es cualquier superficie de suelo que adopte una cierta inclinación con respecto a la horizontal de manera permanente. Los fallos en los taludes están asociados a los movimientos de la masa de suelo que conforman la estructura, pudiendo dar lugar a la pérdida material y de vidas humanas.

Según Armas y Horta (1987) los deslizamientos ocurridos en presas de tierra pueden agruparse en tres categorías de acuerdo al momento de ocurrencia del mismo: durante la construcción, talud aguas abajo durante la operación y talud aguas arriba luego de un desembalse rápido. En esta investigación se consideran los estados de final de la construcción y desembalse rápido, dado que se analizará la modificación de la pendiente sólo en el talud aguas abajo de la presa.

Para definir las características físico-mecánicas de los suelos de la investigación se considera lo planteado por Armas y Horta (1987), dado que la presa analizada tiene una altura de cortina de $22 \mathrm{~m}$, en este caso los parámetros de resistencia efectivos se obtendrán mediante el ensayo lento de corte directo, pudiendo realizarse el ensayo bajo carga controlada o bajo deformación controlada. Estos valores de cohesión y ángulo de fricción interna efectivos se combinarán con las presiones de poros correspondientes en cada caso. Por lo que no resulta necesario la realización de ensayos triaxiales.

Comúnmente se refiere la estabilidad de los taludes en términos de un factor de seguridad FS, que se puede obtener partiendo de un determinado análisis matemático. El valor del factor de seguridad se obtiene al realizar la comparación del esfuerzo cortante de falla con la resistencia cortante del suelo y debe cumplir siempre como requisito, el ser mayor o igual al valor del factor que se establece por norma o se fija en proyecto. Según Sanhueza y Rodríguez (2012), el factor de seguridad expresa la reducción de la resistencia a cortante del suelo para que se produzca el deslizamiento a lo largo de la superficie de falla, y representa la determinación cuantitativa del índice de riesgo de que esta ocurra.

\section{Obtención del Factor de Seguridad}

Para el análisis de la estabilidad, se plantea la utilización del Método de Elementos Finitos MEF, el cual resulta una de las herramientas numéricas más utilizadas en la actualidad para resolver problemas de ingeniería. Se asume un dominio discretizado en subdominios de elementos, donde los elementos son definidos en un número discreto de puntos, llamados nodos, que conectan entre sí a los elementos; y sobre estos nodos se materializan las incógnitas principales del problema que se pretende analizar. El paquete computacional GeoStudio 2012 (www.geoslope.com) presenta la posibilidad de calcular el factor de seguridad FS en presas de tierra a partir de la implementación del MEF, usando un análisis parental entre 
las herramientas SIGMA/W y SLOPE/W, considerando la siguiente fórmula:

$$
F S=\frac{\sum F_{r}}{\sum F_{m}}
$$

donde $F_{r}$ es la resistencia cortante total disponible y $F_{m}$ es la fuerza cortante total movilizada a lo largo de la superficie de falla. La fuerza de resistencia disponible de cada dovela se calcula multiplicando la resistencia al corte del suelo en el centro de la base de la dovela por la longitud de la base. Por lo tanto, a partir de la forma modificada de la ecuación de Mohr-Coulomb para un suelo parcialmente saturado, la fuerza de resistencia disponible viene dada por:

$$
F_{r}=\tau \beta=\left\{c^{\prime}+\left(\sigma_{n}-u_{a}\right) \tan \phi^{\prime}+\left(u_{a}-u_{w}\right) \tan \phi^{b}\right\} \beta
$$

donde $\tau$ es el corte efectivo del suelo en el centro de la base de una dovela, $\beta$ es la longitud de la base de una dovela y $\sigma_{n}$ es la tensión normal en el centro de la base de una dovela. De manera similar, la fuerza cortante total movilizada a lo largo de la superficie de falla de cada dovela se calcula multiplicando la tensión tangencial movilizada $\tau_{m}$ en el centro de la base de la dovela y la longitud de la base $\beta$.

$$
F_{m}=\tau_{m} \beta
$$

Tanto la tensión normal $\sigma_{n}$ como la tensión de tangencial movilizada $\tau_{m}$ son valores obtenidos de un análisis en SIGMA/W. Por lo tanto, las ecuaciones para calcular los factores de estabilidad son lineales; es decir, no se requiere una iteración para establecer los factores de estabilidad como en los métodos de equilibrio límite. SLOPE/W plantea dos ecuaciones fundamentales de equilibrio para obtener el factor de seguridad, una ecuación de equilibrio de momentos y una ecuación de equilibrio de fuerzas.

$$
\begin{aligned}
& E_{m}=\frac{\sum\left(c^{\prime} \beta R+\left[N-u_{w} \beta \frac{\tan \phi^{b}}{\tan \phi^{\prime}}-u_{a} \beta\left[1-\frac{\tan \phi^{b}}{\tan \phi^{\prime}}\right]\right] R \tan \phi\right)}{\sum W x-\sum N f+\sum k W e \pm \sum D d \pm \sum A a} \\
& E_{f}=\frac{\sum\left(c^{\prime} \beta R \cos \left[N-u_{w} \beta \frac{\tan \phi^{b}}{\tan \phi^{\prime}}-u_{a} \beta\left[1-\frac{\tan \phi^{b}}{\tan \phi^{\prime}}\right]\right] \tan \phi \cos \alpha\right)}{\sum N \operatorname{sen} \alpha+\alpha \sum k W e-\sum D \cos \omega \pm \sum A}
\end{aligned}
$$

Donde $W$ es el peso total de una dovela de ancho $\beta$ y altura $h, N$ es la fuerza normal total sobre la base de la dovela, $D$ es un punto externo de carga, $k W$ es la carga sísmica horizontal aplicada a través del centroide de cada corte, $R$ es el radio para una superficie de deslizamiento circular o el brazo de momento asociado con la fuerza de corte movilizada para cualquier forma de superficie de deslizamiento, $f$ es la desviación perpendicular de la fuerza normal desde el centro de rotación o desde el centro de los momentos, $x$ es la distancia horizontal desde la línea central de cada corte hasta el centro de rotación o el centro de los momentos, $e$ es la distancia vertical desde el centroide de cada dovela hasta el centro de rotación o el centro de los momentos, $d$ es la distancia perpendicular desde una carga puntual al centro de rotación o al centro de los momentos, $a$ es la distancia perpendicular desde la fuerza de agua externa resultante hasta el centro de rotación o el centro de momentos, $A$ son las fuerzas de agua externas resultantes, $\omega$ es el ángulo de la carga puntual desde la horizontal y $\alpha$ es el ángulo entre la tangente al centro de la base de cada dovela y la horizontal. La fuerza normal total sobre la base de una dovela $N$ se obtiene como:

$$
N=\frac{W+\left(X_{R}-X_{L}\right)-\frac{\left[c^{\prime} \beta \operatorname{sen} \alpha+u_{a} \beta \operatorname{sen} \alpha\left(\tan \phi^{\prime}-\tan \phi^{b}\right)+u_{W} \beta \operatorname{sen} \alpha \tan \phi^{b}\right]}{F}+D \operatorname{sen} \omega}{\cos \alpha+\frac{\operatorname{sen} \alpha \tan \phi^{\prime}}{F}}
$$

donde $F$ es el factor de seguridad y $X$ son las fuerzas verticales de corte transversal. Los subíndices L y R definen los lados izquierdo y derecho respectivamente. La fórmula (10) se puede utilizar para suelos tanto saturados como no saturados. Para la mayoría de los análisis, la presión de aire en los poros $u_{a}$ se puede considerar cero.

El objetivo fundamental de esta investigación es analizar el comportamiento del factor de seguridad en el talud aguas abajo, para una presa de $22 \mathrm{~m}$ de altura, homogénea, conformada por tres suelos con clasificación $\mathrm{CH}$ y de los cuales se tienen dos curvas características correspondientes a dos grados de compactación. Se plantean tres posibles cimentaciones, todas de tipo arena arcillosa y se analizarán los estados de final de la construcción y operación de la presa. Se observará la dependencia del factor de seguridad del talud aguas abajo del grado de compactación y succión del suelo.

\section{Metodología}

Para la elaboración del modelo, se deben tener en cuenta las consideraciones de diseño para las presas de tierra en Cuba. A partir de estudios realizados por el Instituto Nacional de Recursos Hidráulicos INRH, de las secciones transversales típicas proyectadas en todas las presas del 
país, se presenta el modelo inicial de una presa de tierra de $22 \mathrm{~m}$ de altura, homogénea, sin berma y con prisma de drenaje, con características geométricas correspondientes a lo propuesto por Armas y Horta (1987), como se muestra en la Tabla 1.

Tabla 1: Características geométricas del modelo

\begin{tabular}{|c|c|c|c|}
\hline $\begin{array}{c}\text { Altura del } \\
\text { modelo, } \mathrm{m}\end{array}$ & $\begin{array}{c}\text { Pendiente aguas } \\
\text { arriba }\end{array}$ & $\begin{array}{c}\text { Pendiente } \\
\text { aguas abajo }\end{array}$ & $\begin{array}{c}\text { Ancho de } \\
\text { corona, } \mathrm{m}\end{array}$ \\
\hline 22 & $1: 2.5$ & $1: 2$ & 5 \\
\hline
\end{tabular}

En el caso del prisma de drenaje, éste mantiene las mismas pendientes que los taludes en el modelo geométrico y una altura de 7 m, pues según Armas y Horta (1987) la altura del prisma en la práctica oscila entre un 5 y un $20 \%$ de la altura de la cortina de la presa. El modelo geométrico se muestra en la Figura 1.

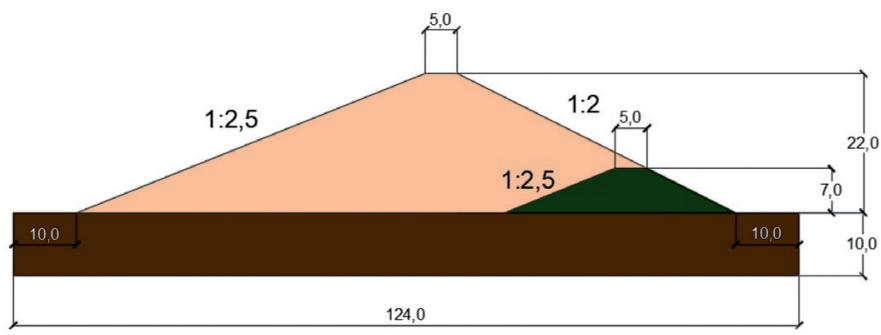

Figura 1: Geometría del modelo de presa de tierra, las dimensiones de altura y ancho en $\mathrm{m}$

Para definir los materiales que conformarán la cortina de la presa se consideraron tres suelos con clasificación $\mathrm{CH}$ (arcilla de alta compresibilidad), cada uno de los cuales presenta dos curvas características obtenidas para diferentes relaciones de vacíos iniciales, los datos y las curvas características de los suelos fueron extraídas de investigaciones previas (Tristá, 2015; Rodríguez, 2017). La relación de coeficiente de permeabilidad horizontal y vertical establecida es de $k_{\mathrm{x}}=12 k_{\mathrm{y}}$ (Armas y Horta, 1987), para respetar el comportamiento de la permeabilidad de los suelos en la naturaleza. Las características de los suelos de la cortina se muestran en la Tabla 2.

A partir del ensayo de granulometría se obtuvieron las curvas granulométricas mostradas en la Figura 2.

Para obtener las humedades de compactación de las muestras remoldeadas se realizó el ensayo Proctor Estándar, obteniéndose los resultados que se muestran en la Figura 3. El remoldeo se realizó para la rama húmeda de la curva de compactación resultante.

Tabla 2: Características de los suelos de la cortina

\begin{tabular}{|l|c|c|c|}
\hline $\begin{array}{l}\text { Características de los suelos de la } \\
\text { cortina }\end{array}$ & Suelo A & Suelo B & Suelo C \\
\hline Peso unitario húmedo $\gamma, \mathrm{kN} / \mathrm{m}^{3}$ & 19.50 & 17.52 & 16.67 \\
\cline { 2 - 4 } & 18.53 & 14.75 & 14.03 \\
\hline Gravedad específica, $G_{\mathrm{s}}$ & 2.82 & 2.72 & 2.72 \\
\hline $\begin{array}{l}\text { Ángulo de fricción interna } \\
\text { efectivo } \phi^{\prime},\end{array}$ & 14.5 & 33.4 & 22 \\
\hline Cohesión efectiva $c^{\prime}, \mathrm{kPa}$ & 49.6 & 3.4 & 29 \\
\hline $\begin{array}{l}\text { Permeabilidad saturada horizontal } \\
k_{\mathrm{x}}, \mathrm{m} / \mathrm{s}\end{array}$ & $4.80 \cdot 10^{-8}$ & $1.03 \cdot 10^{-7}$ & $8.00 \cdot 10^{-8}$ \\
\hline Módulo de elasticidad $E, \mathrm{MPa}$ & 23 & 20 & 22 \\
\hline $\begin{array}{l}\text { Índice de compresibilidad } \\
\text { volumétrica } M_{\mathrm{v}}, 1 / \mathrm{kPa}\end{array}$ & $9.80 \cdot 10^{-5}$ & $8.47 \cdot 10^{-4}$ & $1.00 \cdot 10^{-5}$ \\
\hline Coeficiente de Poisson $\mu$ & 0.33 & 0.33 & 0.33 \\
\hline
\end{tabular}

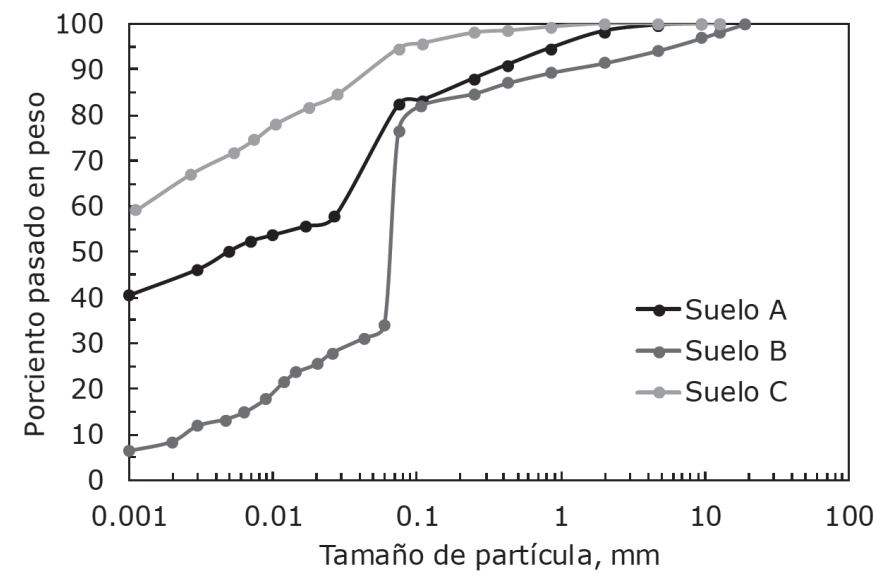

Figura 2: Curvas granulométricas de los suelos (Tristá, 2015; Rodríguez, 2017)

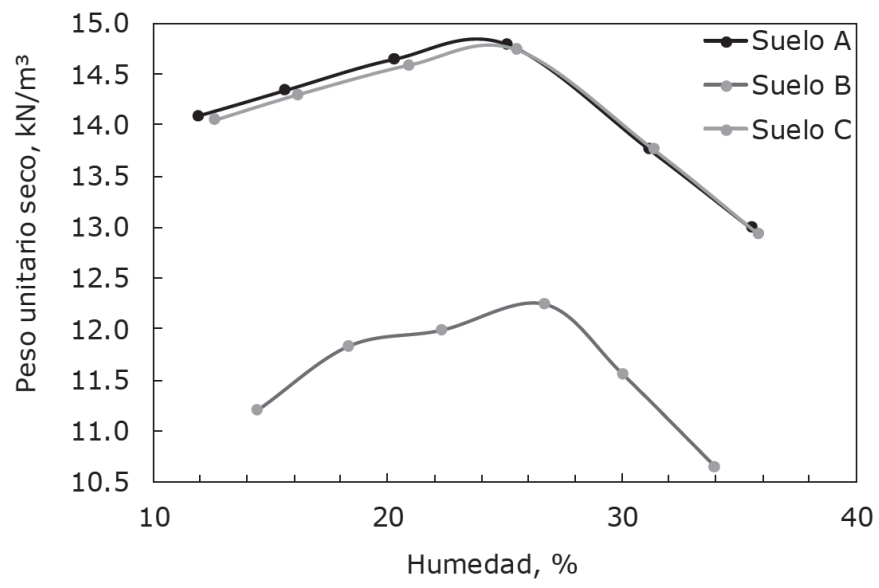

Figura 3: Curvas de compactación obtenidas en los ensayos Proctor Estándar (Tristá, 2015; Rodríguez, 2017) 
En la cimentación se utilizarán tres suelos arenosos de clasificación SC (arena arcillosa) como se muestra en la Tabla 3, esto posibilitará establecer cinco combinaciones.

Tabla 3: Características de los suelos de la cimentación

\begin{tabular}{|c|c|c|c|}
\hline $\begin{array}{l}\text { Características de los suelos de la } \\
\text { cimentación }\end{array}$ & $\mathrm{C}_{1}$ & $\mathrm{C}_{2}$ & $\mathrm{C}_{3}$ \\
\hline Peso unitario húmedo $\gamma, \mathrm{kN} / \mathrm{m}^{3}$ & 17.5 & 18.5 & 19.5 \\
\hline Ángulo de fricción interna $\phi{ }^{\prime},{ }^{\circ}$ & 35 & 37 & 39 \\
\hline Cohesión $c^{\prime}, \mathrm{kPa}$ & 10 & 15 & 20 \\
\hline $\begin{array}{l}\text { Permeabilidad saturada horizontal } \\
k_{\mathrm{x}}, \mathrm{m} / \mathrm{s}\end{array}$ & \multicolumn{3}{|c|}{$10^{-6}$} \\
\hline Módulo de elasticidad $E, \mathrm{MPa}$ & 65 & 75 & 85 \\
\hline $\begin{array}{l}\text { Índice de compresibilidad } \\
\text { volumétrica } M_{\mathrm{v}}, 1 / \mathrm{kPa}\end{array}$ & \multicolumn{3}{|c|}{$10^{-5}$} \\
\hline Coeficiente de Poisson $\mu$ & \multicolumn{3}{|c|}{0.4} \\
\hline
\end{tabular}

En el drenaje se considera una grava de alta permeabilidad, según lo planteado por Coduto (2001) y Das (2001) como se muestra en la Tabla 4.

Tabla 4: Características del drenaje

\begin{tabular}{|l|c|}
\hline Peso unitario húmedo $\gamma, \mathrm{kN} / \mathrm{m}^{3}$ & 21 \\
\hline Ángulo de fricción interna $\phi^{\prime},{ }^{\circ}$ & 40 \\
\hline Cohesión $c^{\prime}, \mathrm{kPa}$ & 0 \\
\hline Permeabilidad saturada $k, \mathrm{~m} / \mathrm{s}$ & $10^{-3}$ \\
\hline Módulo de elasticidad $E, \mathrm{MPa}$ & 18 \\
\hline Índice de compresibilidad volumétrica $M_{\mathrm{v}}, 1 / \mathrm{kPa}$ & $10^{-5}$ \\
\hline Coeficiente de Poisson $\mu$ & 0.27 \\
\hline
\end{tabular}

En las Figuras 4a y $4 \mathrm{~b}$ se muestran las curvas características correspondientes al suelo A, obtenidas por el método del papel de filtro para un grado de compactación de $95 \mathrm{y}$ $100 \%$ del peso unitario seco máximo $\gamma_{\mathrm{dmax}}$ obtenido en el ensayo Proctor Estándar (Rodríguez, 2017).

En las Figuras 5a y $5 \mathrm{~b}$ se muestran las curvas características correspondientes al suelo B, obtenidas por el método del papel de filtro para un grado de compactación de 80 y $95 \%$ de $\gamma_{\text {dmax }}$ obtenido en el ensayo Proctor Estándar (Tristá, 2015).

En las Figuras 6a y 6b se muestran las curvas características correspondientes al suelo $\mathrm{C}$, obtenidas por el método del papel de filtro para un grado de compactación de 80 y $95 \%$ de $\gamma_{\text {dmax }}$ obtenido en el Proctor Estándar (Tristá, 2015). a)

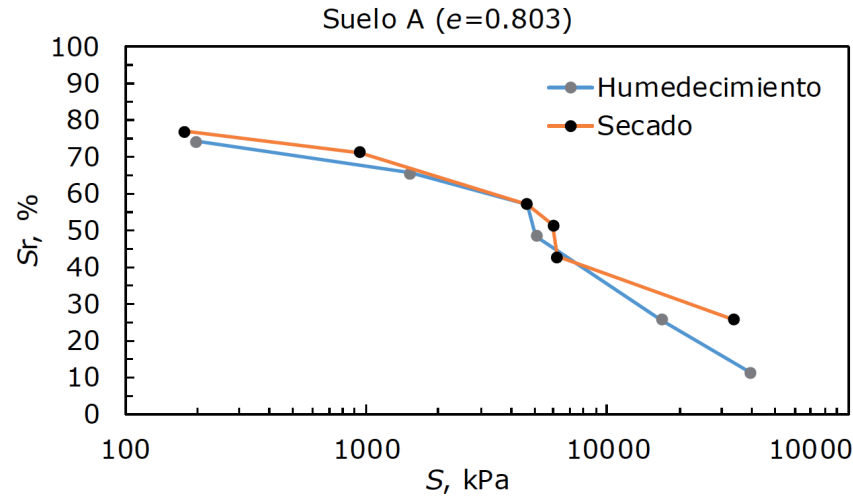

b)

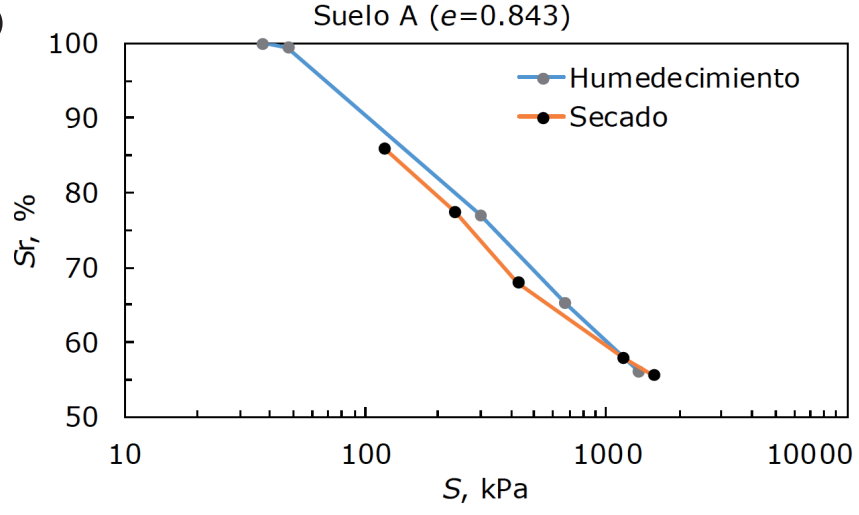

Figura 4: Curva característica del suelo A con: a) razón de vacíos $\mathrm{e}=0.803$ compactada con una humedad de $23 \%$ y b) con $e=$ 0.843 compactada con una humedad de $27 \%$ (Rodríguez, 2017)

a)

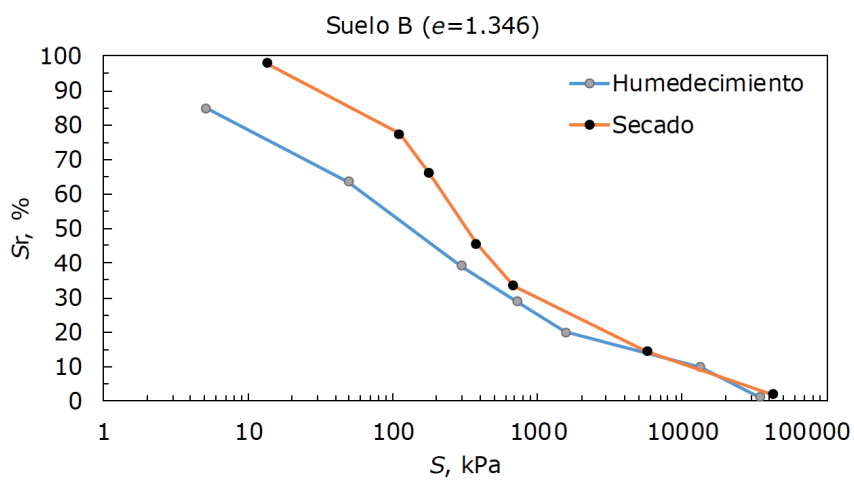

b)

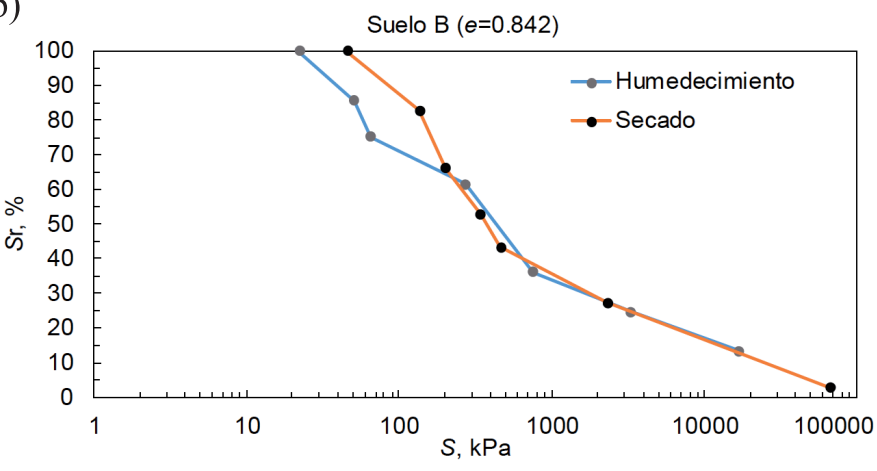

Figura 5: Curva característica del suelo B con: a) $e=1.346$ compactada con una humedad de $26 \%$ y b) con $e=0.842$ compactada con una humedad de 30\% (Tristá, 2015) 
a)

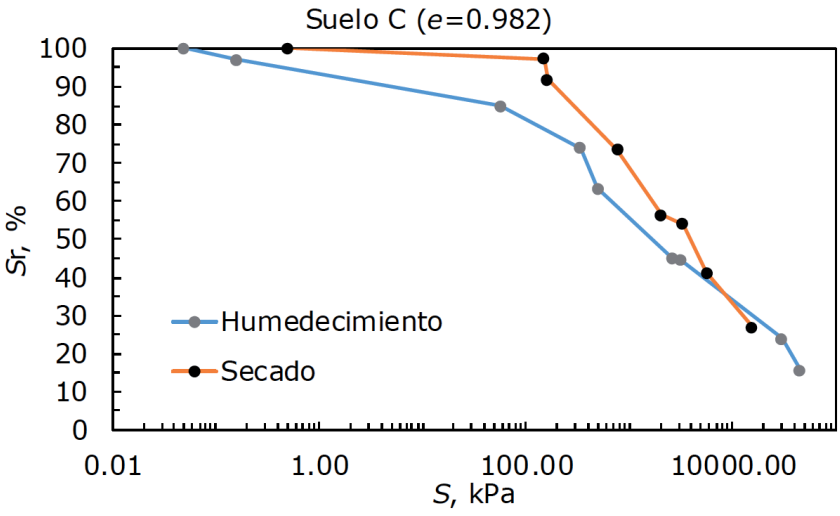

b)

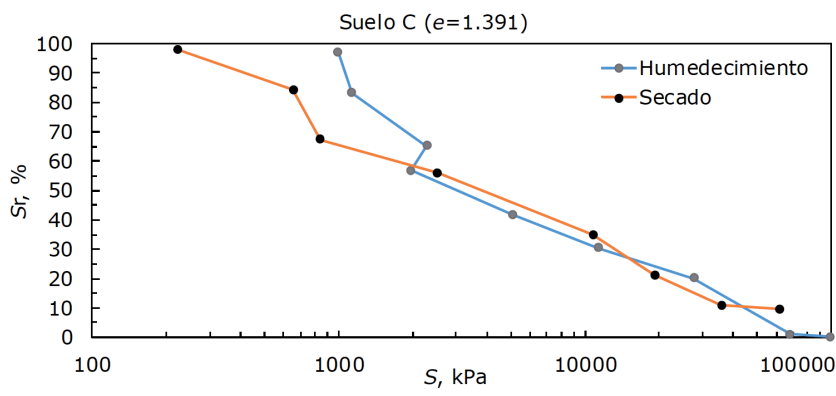

Figura 6: Curva característica del suelo C con: a) $e=0.982$ compactada con una humedad de $27 \%$ y b) con $e=1.391$ compactada con una humedad de 30\% (Tristá 2015)

Los deslizamientos en una presa de tierra están vinculados directamente al nivel de agua que tenga la misma en función del momento en el que estos se produzcan. En esta investigación se analizan los estados de: final de la construcción y durante la operación bajo diferentes condiciones de saturación de los suelos de la cortina. Los niveles de agua se muestran en las Figuras $7 \mathrm{a}$ y $7 \mathrm{~b}$.

a)

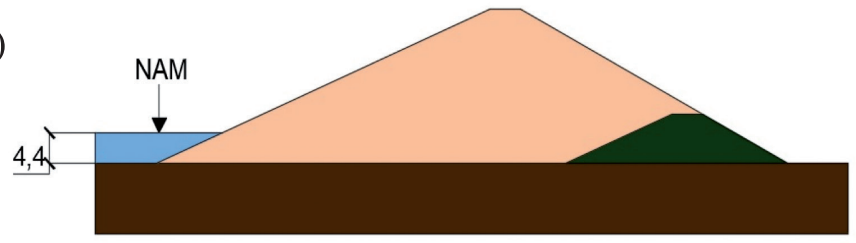

b)

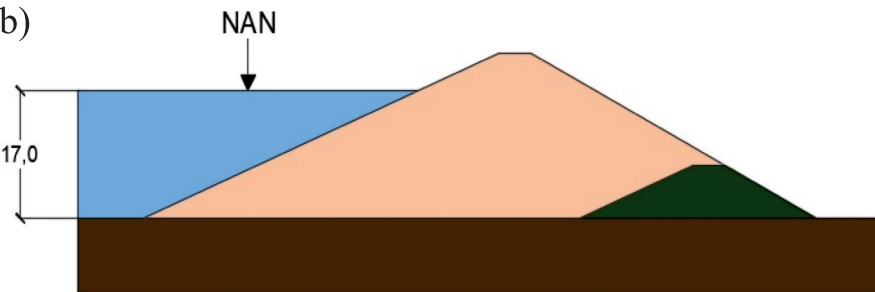

Figura 7: a) Final de la construcción con el nivel de aguas muertas y b) operación con el nivel de aguas normales

\section{Resultados y discusión}

Se parte de obtener en el SIGMA/W la malla más adecuada para cada modelo sin considerar la presencia de agua dentro del embalse ni la succión, por lo que el grado de compactación del material de la cortina será patente sólo por la modificación del peso específico húmedo del suelo de la cortina para cada caso. A partir de esta calibración se obtuvieron los resultados para los tres suelos con las tres cimentaciones, para un total de 9 combinaciones. De forma general, según los valores de asentamientos y esfuerzos máximos en la corona se plantea una distribución de malla de elementos finitos conformada por cuadrados y triángulos con $0.6 \mathrm{~m}$ de longitud, mostrada en la Figura 8.

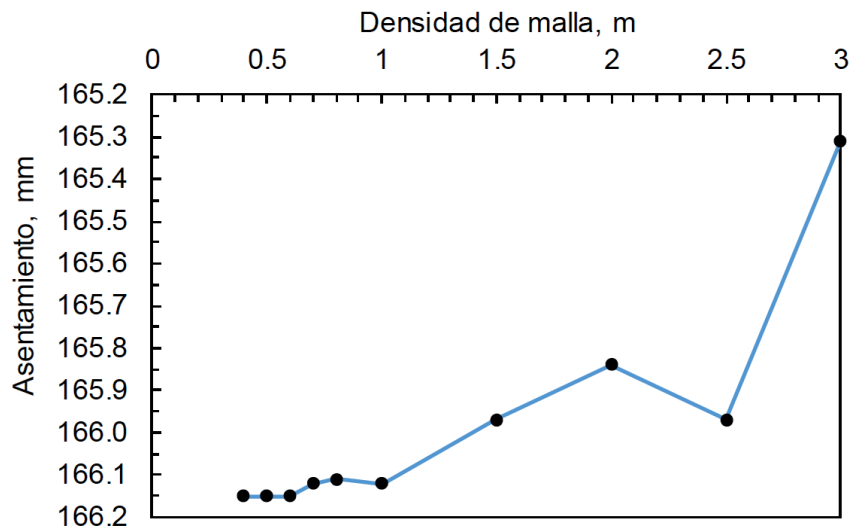

Figura 8: Calibración de la malla de elementos finitos en función de los asentamientos

En cuanto a los asentamientos totales que se producen en la cortina de la presa, para la densidad de malla de $0.6 \mathrm{~m}$ con cada grado de compactación (Gc) analizados, son los que se muestran en las Figuras 9, 10 y 11, para cada suelo considerado.

Suelo A

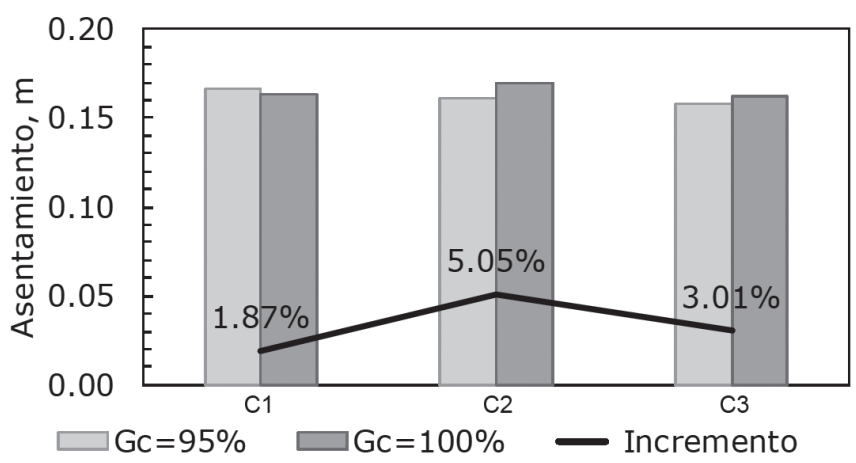

Figura 9: Asentamientos e incrementos del factor de seguridad para el suelo A compactado con 95 y $100 \%$ del peso unitario máximo alcanzado en el ensayo Proctor Estándar 
Suelo B

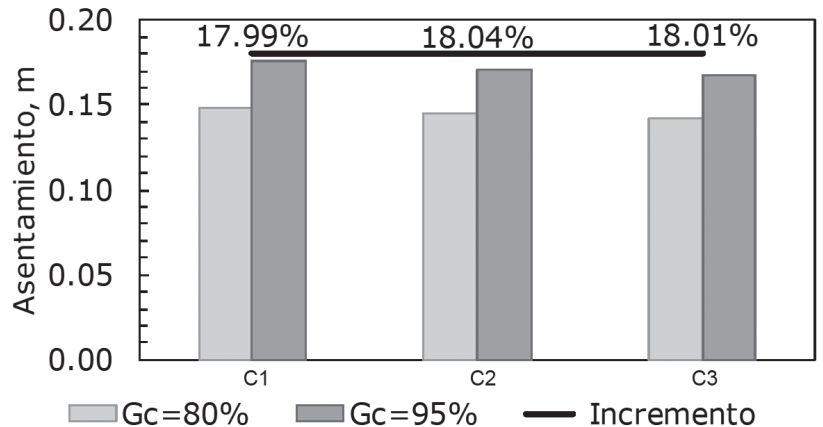

Figura 10: Asentamientos e incrementos del factor de seguridad para el suelo B compactado con 80 y $95 \%$ del peso unitario máximo alcanzado en el ensayo Proctor Estándar

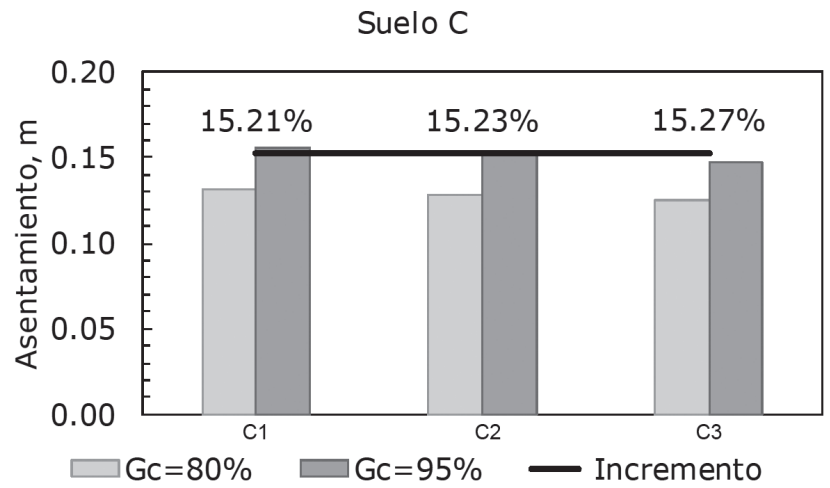

Figura 11: Asentamientos e incrementos del factor de seguridad para el suelo $\mathrm{C}$ compactado con 80 y $95 \%$ del peso unitario máximo alcanzado en el ensayo Proctor Estándar

Se observa en las Figuras 9, 10 y 11 que el incremento en los asentamientos es de 5\% para el suelo A, donde se han utilizado pesos unitarios correspondientes al 95 y $100 \%$ del grado de compactación, mientras que para el suelo B existen incrementos de hasta $18 \%$ y para el suelo $\mathrm{C}$ de $15 \%$, donde las energías de compactación varían entre el 80 y el 95\%.

En el proceso de compactación de suelos influye la humedad de compactación, tipo de suelo, método de compactación que depende de la granulometría, volumen de suelo compactado y energía de compactación (Juárez y Rico, 1996; Llique, 2015). Según Llique (2015) el contenido de humedad es importante durante la compactación, ya que depende de la cantidad de agua en la masa del suelo para que las partículas y grupo de partículas minerales puedan reordenarse bajo una determinada energía de compactación, además, condiciona las propiedades del suelo.

Cada suelo puede tener varias curvas características, en correspondencia con el grado de compactación con el que se ensaye el mismo. En esta investigación se analiza la independencia de la curva de conductividad hidráulica del grado de compactación alcanzado en la cortina de la presa. Los resultados alcanzados para el estado de final de la construcción se muestran en las Figuras 12, 13 y 14 para cada suelo.

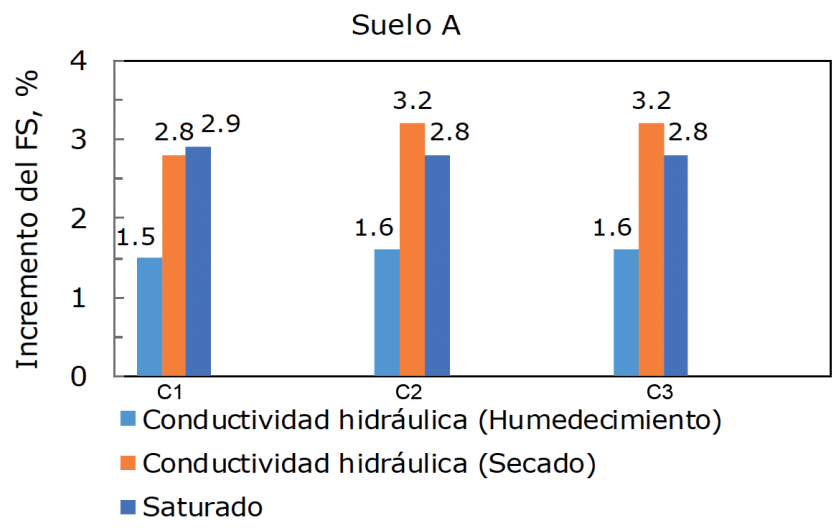

Figura 12: Incremento porcentual del factor de seguridad FS del suelo A al final de la construcción, compactado con 95 y 100\% del peso unitario máximo alcanzado en el ensayo Proctor Estándar

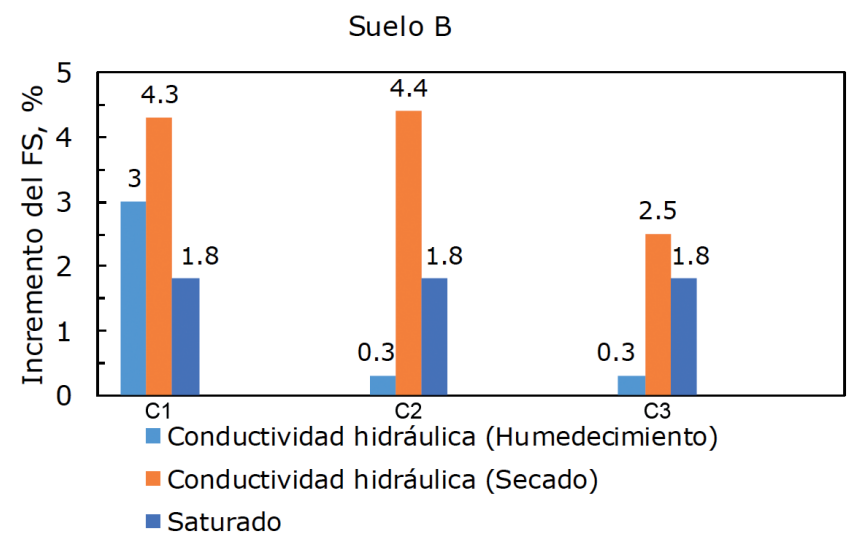

Figura 13: Incremento porcentual del factor de seguridad FS del suelo B al final de la construcción, compactado con 80 y $95 \%$ del peso unitario máximo alcanzado en el ensayo Proctor Estándar

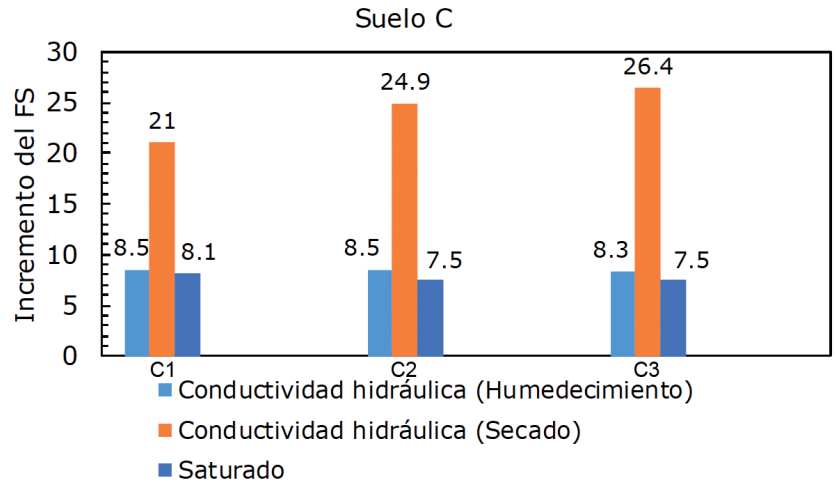

Figura 14: Incremento porcentual del factor de seguridad FS del suelo C al final de la construcción, compactado con 80 y $95 \%$ del peso unitario máximo alcanzado en el ensayo Proctor Estándar 
Como se observa en las Figuras 12, 13 y 14, para los suelos A y B no existe un incremento significativo (mayor que el 5\%) del factor de seguridad FS, al analizar la succión con curvas de conductividad hidráulicas obtenidas para distintos grados de compactación. Para el suelo C, por el contrario, existen incrementos del factor de seguridad de hasta $26 \%$. Por lo tanto, los valores de cohesión y ángulo de fricción interno efectivos, vinculados al grado de compactación, afectan directamente el factor de seguridad. De forma general, las mayores dispersiones se observan para los suelos cuyos grados de compactación se encuentran entre 80 y $95 \%$.

Los resultados correspondientes para todos los casos, en el estado de operación de la presa se muestran en las Figuras 15,16 y 17.

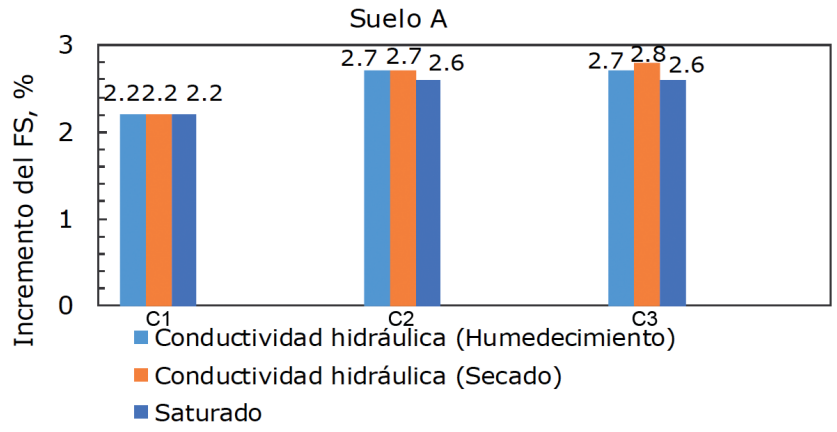

Figura 15: Incremento porcentual del factor de seguridad FS del suelo A en operación, compactado con 95 y $100 \%$ del peso unitario máximo alcanzado en el ensayo Proctor Estándar

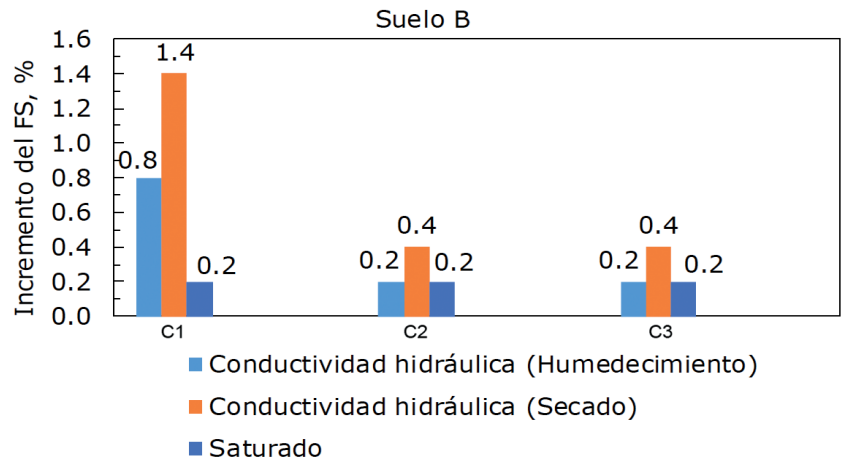

Figura 16: Incremento porcentual del factor de seguridad FS del suelo B en operación, compactado con 80 y $95 \%$ del peso unitario máximo alcanzado en el ensayo Proctor Estándar

Se observa en las Figuras 15, 16 y 17, al igual que para el caso al final de la construcción, que para los suelos A y B no existe, de manera general, un incremento significativo (mayor que el 5\%) del factor de seguridad FS, al analizar la succión con curvas características obtenidas para distintos grados de compactación. Sin embargo, para el suelo C existen incrementos superiores al $5 \%$.

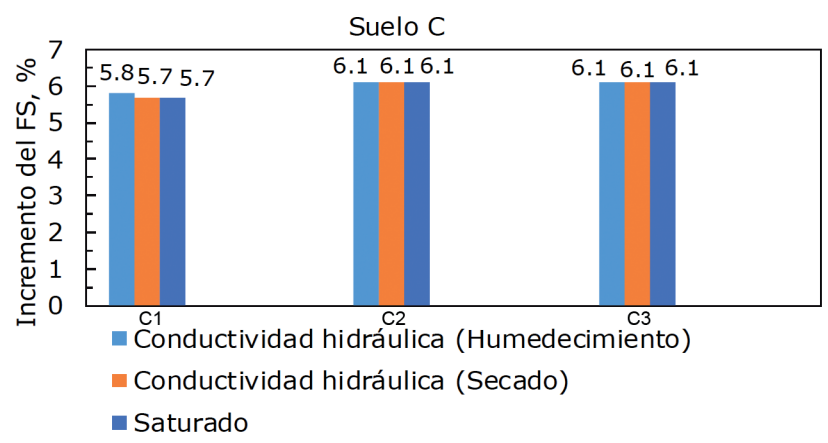

Figura 17: Incremento porcentual del factor de seguridad FS del suelo C en operación, compactado con 80 y $95 \%$ del peso unitario máximo alcanzado en el ensayo Proctor Estándar

Luego de analizados todos los resultados y viendo la variación entre ellos, el resto de la investigación se expone a partir de los resultados correspondientes al $95 \%$ de grado de compactación, dado que para las presas de tierra siempre se proyecta una compactación superior al $90 \%$ del Proctor Estándar. El incremento porcentual entre los factores de seguridad en estado parcialmente saturado, respecto al estado saturado, se muestran en las Figuras 18 y 19 para las nueve combinaciones estudiadas.

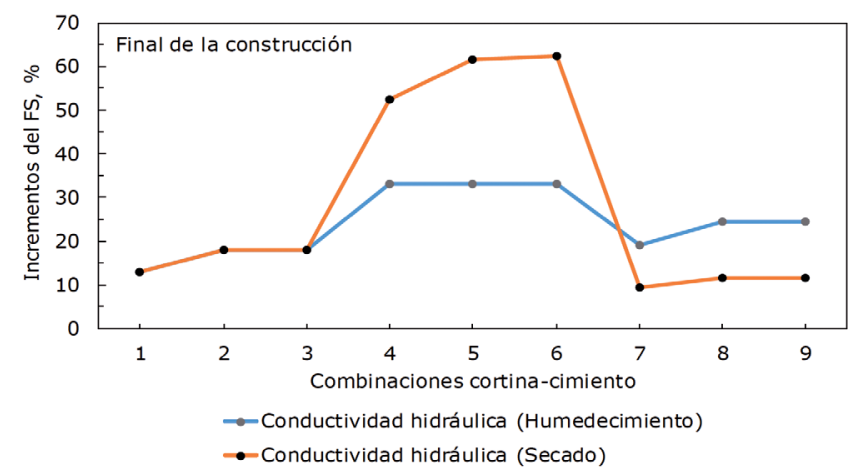

Figura 18: Incrementos del factor de seguridad FS entre los estados saturado y parcialmente saturado al final de la construcción para las nueve combinaciones cortina-cimiento planteadas

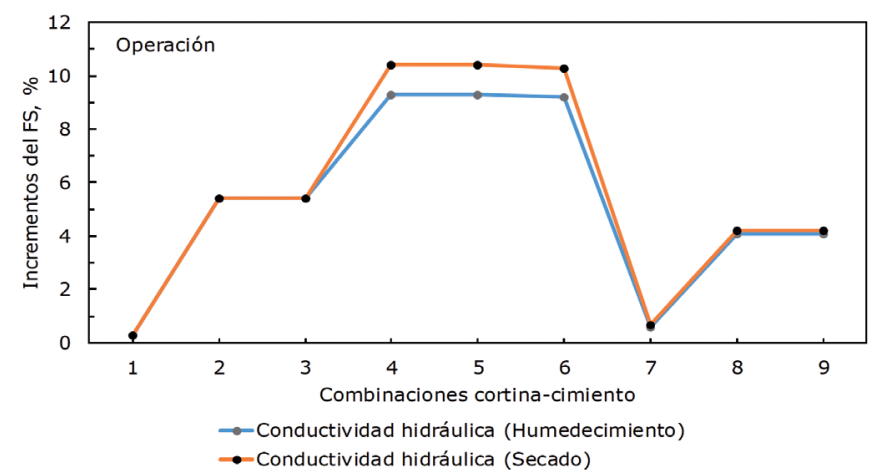

Figura 19: Incrementos del factor de seguridad FS entre los estados saturado y parcialmente saturado en operación para las nueve combinaciones cortina-cimiento planteadas 
En las Figuras 18 y 19 se exponen incrementos entre un 12 y un $60 \%$ para el análisis al final de la construcción, estos datos muestran una amplia dispersión pues, aunque los tres suelos presentan clasificación $\mathrm{CH}$, los valores de cohesión y ángulo de fricción interno son muy variables entre un suelo y otro. Para el análisis en operación, los incrementos se reducen significativamente, quedando entre un 0.3 y un $10 \%$, dado que aumenta el volumen de suelo saturado en todos los casos. En ambos estados de carga, el suelo que presenta los mayores incrementos es el suelo $\mathrm{B}$, que es el caso donde la cohesión es menor y al ángulo de fricción interna es mayor.

\section{Conclusiones}

Los asentamientos generales que se producen en la cortina de la presa oscilan entre 12 y $17 \mathrm{~cm}$, lo que es subsanado durante el proceso de construcción de la obra. Cuando el suelo es compactado con pesos unitarios secos $\gamma_{d}$ entre 95 y $100 \%$ del grado de compactación del Proctor Estándar no existen incrementos significativos de los asentamientos. Sin embargo, cuando las energías de compactación varían entre el 80 y el 95\%, estos incrementos aumentan significativamente.

Para los estados de final de la construcción y operación existen variaciones superiores al 5\% para algunas de las combinaciones analizadas al considerar la influencia de la compactación, a partir de la relación de vacíos $e$, con los valores de succión en cada caso. A partir de estos resultados puede establecerse que, aunque un mismo suelo tiene tantas curvas características como grados de compactación deseen analizarse, en el caso de las presas de tierra con suelos parcialmente saturados, se recomienda utilizar aquellas curvas características experimentales o sus modificaciones, que hayan sido obtenidas para valores de compactación superiores al 95\% del Proctor Estándar.

En el análisis de la estabilidad, resulta significativo considerar el estado de carga en el que se encuentra la presa en el momento del análisis. En los resultados obtenidos se observa que, al incluir la curva de conductividad hidráulica del suelo, el estado de final de la construcción presenta valores superiores al $10 \%$ en todos los casos, mientras que, en operación, estos incrementos se reducen a menos del $5 \%$, lo que puede definir la inclusión o no de las leyes de la mecánica de suelos parcialmente saturado en la obtención del factor de seguridad en la estabilidad de taludes en presas de tierra.

\section{Referencias}

Alanís, A.O. (2012). Deformación volumétrica en suelos no saturados. Tesis de doctorado, Universidad Autónoma de Querétaro, México

Armas, R. y Horta, E. (1987). Presas de Tierra. Ingeniería Hidráulica. Instituto Superior Politécnico José Antonio Echeverría (Cujae), La Habana, Cuba

Coduto, D.P. (2001). Foundation Design: Principles and Practices. Second Edition, Prentice Hall, USA

Das, B.M. (2001). Fundamentos de Ingeniería Geotécnica. Thomson Learning, México

Fredlund, D.G. (2003). Implementación de la mecánica del suelo parcialmente saturado en la práctica de la ingeniería geotécnica. Editorial Litoimpresos, Medellín, Colombia

Fredlund, D.G. and Rahardjo, H. (1993). Soil mechanics for unsaturated soils. John Wiley and Sons, New York, USA

Fredlund, D.G., Morgenstern, N.R. and Widger, R.A. (1978). The shear strength of unsaturated soils. Canadian Geotechnical Journal 15(3), 313-321

Juárez, E. y Rico, A. (1996). Mecánica de Suelos. Tomo 1: Fundamentos de la Mecánica de Suelos. Limusa, México

Llique, R.H. (2015). Influencia de la humedad de compactación en el comportamiento volumétrico de los suelos arcillosos. Tesis de doctorado, Universidad Nacional de Trujillo, México

Mendoza Promotor, J.A. (2018). Influencia de las propiedades no saturadas del suelo en los análisis numéricos de fujo de agua y estabilidad de taludes. Tesis de maestría, Universidad Nacional Autónoma de México

Moffat, R. (2015). Aplicación de un modelo hidromecánico de erosión interna asociado al esfuerzo en la fracción fina del suelo. Obras y Proyectos 17, 39-44

Molina, S., Álvarez, O. y González Y. (2017). Análisis y solución de la patología en presas de Cuba: Estado del arte. II Foro Internacional de Control de la Calidad y Patología de las Construcciones COPACON, La Habana, Cuba

Musso, J. y Suazo, G. (2019). Determinación de la curva de retención de agua para relaves multimetálicos de la industria minera de Chile. Obras y Proyectos 25, 22-29 
Rodríguez, C.M. (2017). Evaluación del comportamiento tensodeformacional en suelos parcialmente saturados con problemas de inestabilidad volumétrica. Tesis de maestría, Universidad Central Marta Abreu de Las Villas, Cuba

Sanhueza, C. y Rodríguez, L. (2012). Análisis comparativo de métodos de cálculo de estabilidad de taludes finitos aplicados a laderas naturales. Revista de la Construcción 12(1), 17-29

Tristá, J.G. (2015). Estudio del comportamiento tensodeformacional de suelos parcialmente saturados en Cuba. Tesis de doctorado, Universidad Central Marta Abreu de las Villas e Instituto Superior Politécnico José Antonio Echeverría, Cuba van Genuchten, M.Th. (1980). A closed-form equation for predicting the hydraulic conductivity of unsaturated soils. Soil Science Society of America Journal 44(5), 892 - 898

Vanapalli, S.K., Fredlund, D.G., Pufahl, D.E. and Clifton, A.W. (1996). Model for the prediction of shear strength with respect to soil suction. Canadian Geotechnical Journal 33(3), 379-392 